\title{
Article
}

\section{Atypical saccadic scanning in autistic spectrum disorder}

Benson, Valerie, Piper, Jenna and Fletcher-Watson, Sue

Available at http://clok.uclan.ac.uk/28071/

Benson, Valerie ORCID: 0000-0002-0351-4563, Piper, Jenna and FletcherWatson, Sue (2009) Atypical saccadic scanning in autistic spectrum disorder. Neuropsychologia, 47 (4). pp. 1178-1182. ISSN 0028-3932

It is advisable to refer to the publisher's version if you intend to cite from the work. http://dx.doi.org/10.1016/j.neuropsychologia.2008.11.019

For more information about UCLan's research in this area go to http://www.uclan.ac.uk/researchgroups/ and search for < name of research Group>.

For information about Research generally at UCLan please go to http://www.uclan.ac.uk/research/

All outputs in CLoK are protected by Intellectual Property Rights law, including Copyright law. Copyright, IPR and Moral Rights for the works on this site are retained by the individual authors and/or other copyright owners. Terms and conditions for use of this material are defined in the policies page.

\section{CLoK}

Central Lancashire online Knowledge www.clok.uclan.ac.uk

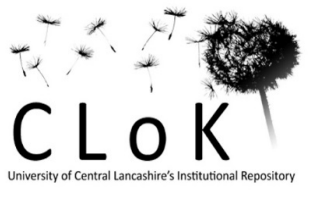


Research Note

Title

Atypical Saccadic Scanning in Autistic Spectrum Disorder.

Valerie Benson ${ }^{1}$, Jenna Piper ${ }^{1} \&$ Sue Fletcher-Watson ${ }^{2}$

${ }^{1}$ School of Psychology, University of Southampton, Southampton, SO17 1BJ, UK.

${ }^{2}$ Institute of Health and Society, Newcastle University, Newcastle, UK

Correspondence should be addressed to Valerie Benson (vb1@soton.ac.uk) 


\begin{abstract}
Saccadic scanning was examined for Typically Developing (TD) adults and those with Autistic Spectrum Disorder (ASD) during inspection of the 'Repin' picture (Yarbus, 1967) under two different viewing instructions: A, Material Instructions ('Estimate the material circumstances of the family'); and B, Social Instructions ('Estimate how long the unexpected visitor has been away'). Proportions of fixations and viewing time on the people and the objects in the scene differed between the two task instructions for TD, but not ASD participants showing that people with ASD did not differentially sample the scene according to top down instruction. One tentative explanation for these findings is that dysfunctional or underdeveloped fronto-parietal feedback systems in ASD, could result in defective saccadic sampling strategies, leading to impairments with cognitive processing in ASD.
\end{abstract}

Key phrases; Saccadic scanning Selective saccadic sampling, Cognitive processing, Autistic Spectrum Disorder (ASD) 


\section{Introduction}

In this paper we report an experiment that examined patterns of eye movements for inspection of the same scene, under two viewing instructions, for two participant groups. It is well established that different types of task systematically influence eye movement metrics (Rayner, 1998; Liversedge \& Findlay, 2000). Patterns of saccades and fixations can reflect not only which features of a display drive the eye movements for a given task (Rayner, 2008) but also how task instruction can modulate saccadic scanning for the same stimulus. For example, when presented with the picture in Figure 1(a), a specific instruction given prior to each viewing results in discrete patterns of saccades and fixations (Yarbus, 1967). This classic work demonstrated how eye movements can reflect the on-line cognitive processing required for a given task in typically developing (TD) individuals. The analysis of eye movements therefore allows us to learn about the influence of different types of visual stimuli, and specific task instructions, on visual and cognitive processing in normal and special populations.

In this paper we examined on-line cognitive processing in participants with Autistic Spectrum Disorder (ASD), by measuring eye movements and manipulating task instruction prior to scene inspection. ASD is characterised by impairments in processing in many domains including social communication (Brenner, Turner \& Muller, 2006) and cognitive function (Russell, 1998). Given the tight relationship between on-line cognitive processing and saccadic scanning, then one might expect that problems in cognitive processing in ASD would be reflected in eye movement patterns for tasks measuring cognitive processing. However, oculomotor control in ASD is still poorly understood (Brenner et al; 2006) and very few studies to date have investigated volitional control of eye-movements when viewing realistic scenes (but 
see Fletcher-Watson, Leekam, Benson, Frank \& Findlay, 2008). Our interest was to investigate whether individuals with ASD selectively sampled a realistic, complex environment in accordance with top down instructions. The main aim was to see if the two participant groups looked at objects and people in the scene differently (more or less frequently and for longer or shorter durations of time) under the two different task instructions, indicating an influence of higher level cognitive processing related to the specific task demands.

\section{Method}

\section{Participants}

ASD participants ( $n=7$, mean age 19yrs), with a formal diagnosis (DSM IV, 1994) were recruited from the European Society for People with Autism (www.espa.org.uk). TD participants $(n=9$, mean age 18yrs) were volunteers from a local sixth form college. All participants were paid and all had normal or corrected to normal vision. Standardised IQ tests (WASI, 1999) were completed on a one-to-one basis prior to eye movement recording. Although the ASD group scored lower on all IQ measures compared to the TD group, they were within the normal range: Full scale IQ (TD 103, ASD 88); Verbal IQ (TD 103, ASD 84) and Performance IQ (TD 102, ASD 95). Ethical approval was obtained from the relevant Psychology Ethics Committee, and all volunteers consented in writing.

\section{Eye movement recording}

Eye position was sampled every $5 \mathrm{~ms}$ using a Fourward-Technologies Dual Purkinjie tracking system with spatial resolution of $10 \mathrm{~min}$ arc. A chin rest and forehead supports were used to stabilize head position. Viewing was binocular but data was recorded for one eye only. Individual participants were calibrated using a nine point matrix that covered the dimensions of the screen (1024 x 768 pixels), each 
point being fixated sequentially. Calibration data was checked on-line prior to trial presentation.

\section{Materials}

A copy of the picture 'Unexpected Return', painted by the Russian artist Ilya Repin in 1884 was downloaded from http://www.abcgallery.com/R/repin/repin46.JPG and converted to a bitmap with a resolution of 1024 x 768 pixels. This was presented, in colour, on a 21 inch monitor, at a distance of 1 metre from the participant's eyes.

\section{Design}

A repeated measures design was employed, with task instruction as a within participants variable, and group as a between participants variable.

\section{Procedure}

Stimuli were displayed on a Philips 21B582BH 21inch monitor at a viewing distance of $1 \mathrm{~m}$. The monitor and the eyetracker were both interfaced with a Philips Pentium III PC that controlled the experiment. Successful calibration preceded the two experimental trials. In house software displayed a central fixation target for a fixed period of 1 second ( $1 \mathrm{deg}$ white cross on a black background) designed to ensure participants fixated the centre of the screen prior to the start of each trial. This was followed by the stimulus display, which initiated eye-movement recording, and which remained visible for 20seconds, followed by a blank screen. Prior to each presentation of the 'Repin' picture participants were given one of two possible inspection instructions; A, Material Instructions ('Estimate the material circumstances of the family'); B, Social Instructions ('Estimate how long the unexpected visitor has been away'). Order was counterbalanced across participants. Following each trial participants were asked to select from one of three options to indicate their response to each viewing instruction. For the material instructions participants were asked to report how well off the family was and could choose from 'wealthy', 'poor', or 
'average' options. For the social instructions, where they estimated how long the unexpected visitor had been away from the family, the options were, 'days', 'weeks', or 'months'. Indirect understanding of the task requirements and the picture content was demonstrated by all participants during the verbal response task.

\section{Data preparation}

We divided the picture into domains of interest (Figure 1b) using colour to demarcate each category for the interest areas. An in-house semi-automated procedure was used to analyse the eye movement data. The start and end of saccades were detected automatically using velocity criterion and all records were inspected individually to check that the program had 'picked up' the saccades. The same software was used to calculate the distribution of fixations falling into each of the interest areas, and for the principal analyses the data were combined for the head and body regions to create a 'people' domain, and for the wall and floor objects to create an 'object' domain for task by group comparison purposes.

\section{Data analyses}

We compared the proportion and duration of fixations falling within the domains of 'people' and 'objects' in a repeated measures ANOVA with task as a within participant variable and group as a between participant variable.

\section{Results}

\section{Proportion of fixations}

The Main Effects were as follows: There were no Main Effects for the people domain analysis. There was a Main Effect of task for the object domain analysis $\mathrm{F}(1,14)=8.35, p .012$, which showed that the proportion of fixations on objects was

greater in task A (mean 17.01\%), compared to task B (mean 8.02\%). There was no significant Main Effect of group. 
What is of greater importance here is that the analysis of the proportion of fixations produced a consistent set of interactions between task and group, illustrated in Figure 1 (c) and reported here in detail. For the people domains an interaction between group and task $\mathrm{F}(1,14)=11.19, p=.005$ showed that the proportion of fixations on people were greater for the TD group for task B (mean 69.03\%), compared to task A (mean 50.92\%); $(\mathrm{t}(8)=-3.52, \mathrm{p}=.008)$, whereas there was no difference in the proportion of fixations on people for the ASD group between the two task instructions, task A (mean 55.80\%), task B (mean 48.62\%); $(\mathrm{t}(6)=1.32, \mathrm{p}=$ .236). For the object domains there was a significant interaction between task and group, $\mathrm{F}(1,14)=10.16, p=.007$ showing that the proportion of fixations on objects was greater for the TD group for task A (mean 22.67\%), compared to task B (mean $6.06 \%) ;(\mathrm{t}(8)=4.58, \mathrm{p}=.002)$, whereas there was no difference in the proportion of fixations on objects for the ASD group between the two task instructions task A (mean 9.73\%), task B (mean 10.54\%); $(\mathrm{t}(6)=-0.20, \mathrm{p}=.848)$. Task instruction clearly modulated saccadic scanning for TD but not ASD participants.

\section{Proportion of viewing time}

The Main Effects were as follows: There were no Main Effects for the people domain analysis. There was a Main Effect of task for the object domain analysis $\mathrm{F}(1,14)=8.63, p .011$, which showed that the proportion of time inspecting objects was greater in task A (mean 16.68\%), compared to task B (mean 7.32\%). There was no significant effect of group.

Thus, the proportion of viewing time spent in each domain showed an identical pattern of results (see Figure 1d) to the fixation analyses. A marginally significant interaction between task and group $\mathrm{F}(1,14)=3.32, p=.090$ showed that the TD group looked longer at the people domain in task B compared to task A (TD 
task A mean 55.40\%, TD task B mean 74.41\%); $(\mathrm{t}(8)=-2.55, \mathrm{p}=.034)$ whereas the ASD group showed no difference in the proportion of time spent looking in the people domain for the two task instructions (ASD task A mean 50.39\%, ASD task B mean $51.16 \%) ;(\mathrm{t}(6)=0.03, \mathrm{p}=.904)$. The object domain analysis also resulted in a significant interaction between task and group $\mathrm{F}(1,14)=8.29, p=.012$ once again showing that the proportion of viewing time differed for the TD group for task instruction, but not the ASD group. Viewing time on objects was greater for the TD group for task A (mean 21.54\%), compared to task B (mean 5.03\%); $(\mathrm{t}(8)=5.09, \mathrm{p}=$ $.001)$, whereas the viewing time on objects for the ASD group between the two task instructions, task A (mean 10.43\%), task B (mean 10.27\%); $(\mathrm{t}(6)=0.03, \mathrm{p}=.974)$ was equivalent.

\section{Head domain analysis}

Heads, and in particular facial expressions, carry important social information (Farah, Drain \& Tanaka, 1998). Consequently these should receive a higher proportion of fixations for Task B than Task A. For the proportion of fixation to heads analysis there was a Main Effect of task $\mathrm{F}(1,14)=7.75, p .015$, which showed that this was greater in task B (mean 39.81\%), compared to task A (mean 30.80\%). There was also a significant Main Effect of group $\mathrm{F}(1,14)=12.30, p .003$, which showed that there was a greater proportion of fixations on heads alone for the TD group (mean 42.16\%) compared to the ASD group (mean 26.50\%). However, an interaction between task and group $\mathrm{F}(1,14)=7.26, p=.017$ showed that the proportion of fixations on heads was greater for the TD group for task B (mean 50.07\%), compared to task $\mathrm{A}($ mean $34.25 \%) ;(\mathrm{t}(8)=-4.37, \mathrm{p}=.002)$, whereas there was no difference in the proportion of fixations on heads for the ASD group between the two task instructions, task B (mean 26.62\%), task A (mean 26.37\%); $(\mathrm{t}(6)=-0.06, \mathrm{p}=.958)$. (see Figure 1c). No significant differences were observed for an analysis of fixations 
on bodies, showing that the interaction between task and group for the 'people' domain reported above was entirely driven by differences between the groups in inspecting heads.

The viewing time analysis resulted in a Main Effect of task $\mathrm{F}(1,14)=9.29, p$ .009 , which showed that the viewing time on heads was greater in task B (mean 46.93\%), compared to task A (mean $34.05 \%$ ). There was also a significant Main Effect of group $\mathrm{F}(1,14)=12.16, p .004$, which showed that more time was spent viewing heads alone for the TD group (mean 49.71\%) compared to the ASD group (mean 28.63\%). The viewing time analysis however did not result in an interaction $\mathrm{F}(1,14)=1.32, p=.270 .(\mathrm{TD}$ task B mean $58.18 \%$, TD task A (mean 41.25\%); ASD task B (mean 32.47\%), ASD task A (mean 24.80\%). This suggests that both groups look longer at the heads in task B compared to task A, and could indicate that ASD participants are paying more attention to the heads in task B. To determine whether this was the case we analysed the mean fixation duration for the individual heads in the scene.

\section{Individual Head(s) analyses}

The heads were numbered as follows; The 'unexpected visitor' was labelled head 1 , the woman in the foreground was labelled head 2, the two women in the doorway were labelled numbers, 3 and 4 from left to right, the female seated at the piano was labelled head number 5 , and the children seated at the table were labelled head 6 and head 7 from left to right. For the mean fixation duration analysis for the heads we divided the total viewing time on each head by the number of discrete fixations upon each head and empty cells were excluded when calculating the means. Any small saccades, greater than half a degree made within a 'head' would be counted as separate fixations. A repeated measures ANOVA was conducted with task and head as within participant variables, and group as a between participants variable. 
Here we report only the significant effects. There was a main effect of 'head', $F(1,6)$ $=3.43, p=.040$. Head means were as follows (head 1, 371ms, head 2, 252ms, head 3, 298ms, head 4, 431ms, head 5, 342ms, head 6, 255ms and head 7, 289ms). Whilst it might be expected that head1, being a central character in the picture, would receive longer fixation durations, it is surprising that head 4 receives the longest fixations. This finding was qualified by a three way interaction between task, head and group $\mathrm{F}(6,84)=2.17, p=.054$ and paired sample t-tests revealed that there was only one significant difference between task A and task B, and that this was for the ASD group. This was for head 3 , $(\mathrm{t}(6)=-3.25, \mathrm{p}=.018)$. All other tests produced $\mathrm{t}$ 's $<2$. It is clear from Figure 1 (e) that there is more variability in the viewing time data for the ASD participants, for some of the heads.

We also checked whether there were any differences in how many of the heads were sampled, across the two groups of participants for the two tasks. For the head skipping analysis we compared the number of heads across the two groups that were never fixated for both viewing inspections, this information was available from the gross measures of viewing time and fixations for the heads only region of interest. This revealed that all participants fixated the head of the man in the picture for both tasks, but overall ASD participants failed to fixate significantly more heads $\mathrm{F}(1,14)=$ $16.78, p=.001$, (mean 2.28) compared to TD participants (mean 0.33).There was no main effect of task $\mathrm{F}(1,14)=.145, p=.001$, and no interaction between task and group $\mathrm{F}(1,14)=.460, p=.509$. The increased head skipping for the ASD group could be due to a problem with disengaging attention, otherwise known as perseveration, and this would be supported by the observation that some of the individual mean fixation durations for the ASD were abnormally long, see Figure 1 (e), one to head 4 being over 2 seconds. 
In summary, the individual heads analysis has shown that there are no differences in mean fixation durations for each head, for each task for the TD group. There was one difference for head 3 for the ASD group, accompanied by high variability and abnormally long fixations in ASD for heads 4 and 5. These results are relevant to the previous analyses. The reason that the interaction for the viewing time analyses to heads did not reach significance was most likely a result of the high variability in viewing time for the ASD group for some of the heads. Indeed, if paired comparisons are made for the proportion of viewing time to heads data set, then the same consistent pattern that has been observed for all other comparisons holds here too (TD task B mean 58.18\%, TD task A (mean 41.25\%); (t $(8)=-2.85, \mathrm{p}=.021)$ ASD task B (mean 32.47\%), ASD task A (mean 24.80\%); $(\mathrm{t}(6)=-1.53, \mathrm{p}=.177)$.

\section{Discussion}

These novel findings imply that saccadic scanning in ASD is not influenced or at least not modulated in the same systematic way by higher level cognitive factors that guide eye movements in TD participants. Comprehension of the different task instructions was tested by verbal questioning following each inspection and all ASD participants responded appropriately and demonstrated a clear understanding of each task requirement. It appears that attention in the ASD group, as measured by saccadic scanning in this paper, is not distributed differentially to the different scene elements according to top down instruction. Although this experiment was not designed as a measure of social attention, the results indicating reduced fixation on faces overall in ASD compared to TD participants does support existing evidence of reduced fixation on social information in ASD (Klin, Jones, Schultz, Volkmar \& Cohen, 2002). Additionally, in a recent review article (Behrmann, Thomas \& Humphreys, 2006) it 
was concluded that there are perceptual processing problems in ASD independent of social function, and we believe that our data support this.

Could frontal lobe dysfunction offer a plausible account for such atypical orienting in ASD? Although such a notion at this stage, with the small sample reported here is speculative, it is known that the frontal lobes have an established role in processes involved in higher cognitive function (Smith \& Jonides, 1999) and the fronto-parietal circuitry is involved in attentional (Corbetta, Miezin, Shulman \& Peterson, 1993), and oculomotor control (Pierrot-Deseillingny, Milea, \& Muri, 2004). Impairments in volitional saccadic control in the antisaccade task, indicative of frontal lobe dysfunction (Munoz \& Everling, 2004) are observed in ASD (Minshew, Luna \& Sweeney, 1999), and consistent with this, when the ASD participants in this study were tested in an antisaccade task, they also made a higher proportion of errors (62.2\%) compared to the TD participants (34.8\%).

Frontal lobe abnormalities in ASD include reduced volume of grey matter (McAlonan, Daly, Kumari, Critchley, van Amelsvoort et al, 2002) increased white matter (Herbert, Zeigler, Makris, Filipek, Kemper, Normandin et al, 2004) and weaker cortical connectivity (Horowitz, Rumsey, Grady \& Rapport, 1988). These aberrations could impact upon the development of feedback systems between the fronto-parietal network and visual processing areas that are necessary for oculomotor control and tasks requiring attentional modulation. Therefore, underdeveloped feedback systems between frontal and parietal lobes may in future be shown to account for, or at least contribute to, deficits in selective saccadic sampling for higher level cognitive tasks in ASD. It is not known how such deficits may impact upon domain general attentional difficulties (Allen \& Courchesne, 2001) or any specific social attention problems (Mundy \& Newell, 2007) in ASD. These questions should 
be addressed through further naturalistic assessments of eye-movements and attention to complex, realistic stimuli (Klin, Jones, Schultz, Volkmar \& Cohen, 2002).

Figure Legends

Figure 1. Stimuli (a) The 'Repin' picture and (b) Regions of interest, which were categorised as people (heads and bodies), objects (on the floor and wall) and background items (doors and windows, walls and ceiling, floor, window panes). (c) Results: Proportion of fixations directed to the people domain (top), the object domain (middle), and the heads only domain (bottom). (d) Proportion of viewing time spent in the people domain (top), the object domain (middle), and the heads only domain (bottom) and (e) Mean fixation durations on the individual heads in the picture, for both groups, for each task instruction. Error bars denote \pm 1 s.e.m.

\section{ACKNOWLEDGMENTS}

We would like to thank Sue Leekam for comments on an earlier draft of this paper, and also Bob Metcalfe for developing the software that enabled the collection and analyses of these data.

\section{References}

Allen, G. \& Courchesne, E.. (2001). Attention function and dysfunction in autism. Frontiers in Bioscience, 6, 105-119.

Behrmann, M., Thomas, C. \& Humphreys, K. (2006). Seeing it differently: visual processing in autism. Trends in Cognitive Sciences. 10, (6) 258-264.

Brenner, L.A., Turner, K.C., \& Muller, R-A. (2006). Eye Movement and Visual Search: Are there Elementary Abnormalities in Autism?. J Autism Dev. Disorder. 37, (7) 1289-1309.

Corbetta, M., Miezin, F.M., Shulman, G.L., Peterson, S.E. (1993). A PET study of visuospatial attention. J. Neuroscience. 13, 1202-1226. 
Diagnostic and Statistical Manual of Mental Disorders $4^{\text {th }}$ edn. (American Psychiatric Association, Washington DC, 1994).

Farah, M.J., Wilson, K.D., Drain, M. \& Tanaka, J.N. (1998). What is "special" about face perception. Psychological Review. 105, 482-498.

Fletcher-Watson, S., Leekam, S., Benson, V., Frank, M.C. and Findlay, J.M. (2008). Eye-movements reveal attention to social information in autism spectrum disorder. Neuropsychologia, forthcoming (doi:10.1016/j.neuropsychologia.2008.07.016) (In Press).

Herbert, M.R., Zeigler, D.A., Makris, N., Filipek, P.A., Kemper, T.L., Normandin, J.J., et al. (2004). Localization of white matter volume increase in autism and developmental language disorder. Annals of Neurology, 55 (4), 530-540.

Horowitz, B., Rumsey, J.M., Grady, C.L.,\& Rapport, S.I. (1988). The cerebral metabolic landscape in autism: intercorrelations of regional glucose utilization. Archives Neurology. 45 749-755.

Klin, A., Jones, W., Schultz, R., Volkmar, F. \& Cohen, D. (2002). Defining and quantifying the social phenotype in autism. American Journal Psychiatry. 159 (6) 895-908.

Klin, A., Jones, W., Schultz, R., Volkmar, F. \& Cohen, D. (2002). Visual Fixation Patterns During Viewing of Naturalistic Social Situations as Predictors of Social Competence in Individuals With Autism. Archives of General Psychiatry. 59, 809816.

Liversedge, S.P. \& Findlay, J.M. Eye movements reflect cognitive processes. (2000). Trends in Cognitive Sciences, 4, 6-14.

McAlonan G.M., Daly E., Kumari V., Critchley H.D., van Amelsvoort T., Suckling J., Simmons A., Sigmundsson T., Greenwood K., Russell A., Schmitz N., Happe F., Howlin P., and Murphy D.G.M. (2002). Brain anatomy and sensorimotor gating in Asperger's syndrome. Brain. 125; (7) 1594-1606.

Minshew, N. A., Luna, B., \& Sweeney, J. A. (1999). Oculomotor evidence for neocortical systems but not cerebellar dysfunction in autism. Neurology. 52, 917-922.

Mundy, P. \& Newell, L. (2007). Curr. Dir. in Psychol. Sci. 16 (5) 269-274.

Munoz, D.P., \& Everling, S. (2004). Look away: the antisaccade task and the voluntary control of eye movement. Nature Review, Neuroscience. 5, 218-228.

Pierrot-Deseilligny, C., Milea, D., Muri, R.M. (2004). Eye movement control by the cerebral cortex. Current Opinion Neurology. 17, 17-25.

Rayner, K. (1998). Eye movements in reading and information processing: 20 years of research. Psychological Bulletin. 124, 372-422. 
Rayner, K. The Thirty-fifth Sir Frederick Bartlett Lecture: Eye Movements and Attention in Reading, Scene Perception, and Visual Search. Quarterly journal of Experimental Psychology (In press).

Russell, J., ed. Autism as an Executive Disorder, Oxford University Press, (1998).

Smith, E.E. \& Jonides, J. (1999). Storage and Executive Processes in the Frontal Lobes. Science. 283, 1657-61.

Wechsler, D. Wechsler abbreviated Scales of Intelligence (WASI) Psychological Corporation, USA, (1999).

Yarbus, A. Eye Movements and Vision. New York: Plenum, (1967). 